\title{
DESIGN AND FULL-CAR TESTS OF ELECTRIC POWER STEERING SYSTEM
}

\author{
Jingbo Zhao *, Long Chen, Haobin Jiang, Limin Niu \\ School of Automobile and Traffic Engineering, Jiangsu University, Zhenjiang, China, 212013 \\ * Corresponding author, Address: P.O. Box 243, School of Automobile and Traffic \\ Engineering, Jiangsu University, 301 Xuefu Road, Zhenjiang, Jiangsu, 212013, P. R. China, \\ Tel: +86-511-82054123, Fax: +86-511-88791221(ext. 2009), Email:zhaojb1128@yahoo.com.cn
}

Abstract: Electric Power Steering (EPS) is a full electric system, which reduces the amount of steering effort by directly applying the output from an electric motor to the steering system. In this paper, the constitutions and its operational mechanism of electric power steering system, and the construction and the equivalent circuit of the DC motor used in EPS were introduced; and the EPS hardware framework based on the ARM was presented and the EPS motor control strategy was designed. The full-car tests were performed and the results confirmed that the system designed was stable and credible, and can meet the requirements of steering performance.

Keywords: $\quad$ automobile, Electric Power Steering, EPS, motor, controller

\section{INTRODUCTION}

Electric power steering (EPS) system has attracted much attention for their advantages. It uses power only when the steering wheel is turned by the driver, it consumes approximately one-twentieth the energy of conventional hydraulic power steering systems and, as it does not contain any oil, it does not pollute the environment both when it is produced and discarded. Additionally, the software built into the EPS controller results in high performance and easy tuning during the development of prototypes of EPS systems (Jiang Haobin et al., 2006).

Fig. 1 shows a vehicle with a column-type EPS in which the reduction gear is located directly under the steering wheel. The EPS system consists of a 


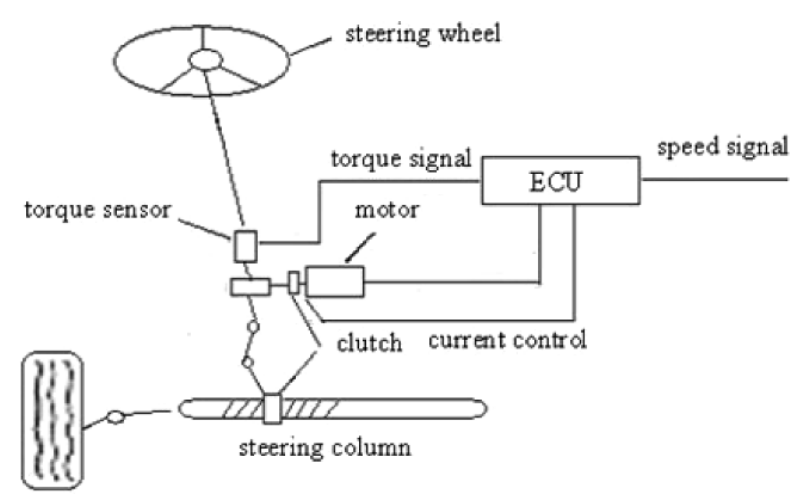

Fig. 1. A columm - type EPS system

torque sensor, which senses the driver's movements of the steering wheel as well as the movement of the vehicle; an ECU, which performs calculations on assisting force based on signals from the torque sensor and vehicle sensor; a motor, which produces turning force according to output from the ECU; and a reduction gear, which increases the turning force from the motor and transfers it to the steering mechanism (Toshinori Tanaka et al., 2003).

The main purpose of electric power steering system is, of course, to provide assist to the driver. This is achieved by the torque sensor, which measures the driver's torque and sends a signal to the controller proportional to this torque. The torque information is processed in the controller and an assist command is generated. This assist command is further modulated by the vehicle speed signal, which is also received by the controller. This command is given to the motor, which provides the torque to the assist mechanism. The gear mechanism amplifies this torque, and ultimately the loop is closed by applying the assist torque to the steering column.

\section{DESIGN OF EPS CONTROLLER}

The motor for EPS is a permanent magnetic field DC motor. Attached to the power steering gear assembly, it generates steering assisting force. This study introduced the motor control strategy, the design of the EPS Electric Control Unit (ECU), and the full-car test system. The full-car tests were performed and the results were analyzed.

\subsection{Motor control strategy}

Fig. 2 illustrates the construction of a DC motor, consisting of a stator, a rotor, and a commutation mechanism (Massachusetts Institute of Technology). The stator consists of permanent magnets, creating a magnetic 
field in the air gap between the rotor and the stator. The rotor has several windings arranged symmetrically around the motor shaft. An electric current applied to the motor is delivered to individual windings through the brushcommutation mechanism, as shown in the figure. As the rotor rotates the polarity of the current flowing to the individual windings is altered. This allows the rotor to rotate continually.

The actual DC motor is not a loss-less transducer, having resistance at the rotor windings and the commutation mechanism. Furthermore, windings may exhibit some inductance, which stores energy. Fig. 3 shows the schematic of the electric circuit, including the windings resistance $\mathrm{R}$ and inductance $\mathrm{L}$.

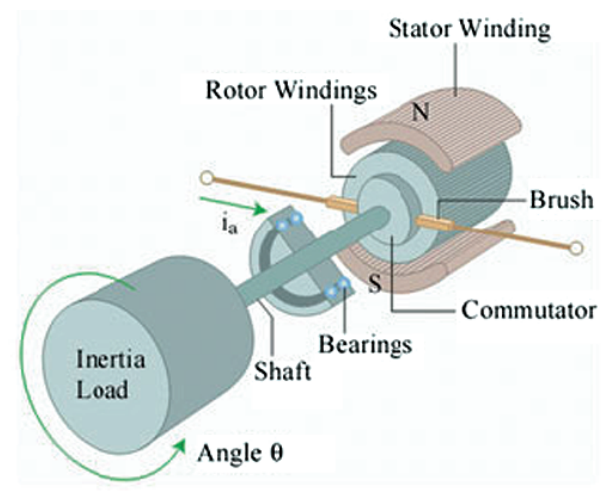

Fig. 2. Construction of DC motor

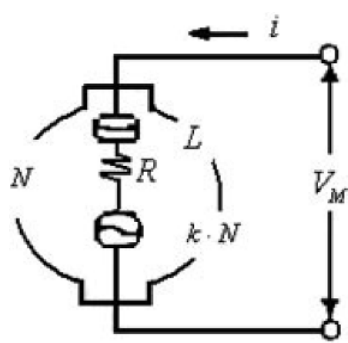

Fig. 3. Equivalent circuit

In the equivalent circuit of the motor, the relationship between the terminal voltage $V_{M}$, the impendance $L$, the resistanc $R$, the induced voltage constant $k$, the revolution speed $N$, the current $i$, and the time $t$, is expressed by the following equation (Ronald K. et al.,1999).

$$
\begin{gathered}
V_{M}=L \cdot(d i / d t)+R \cdot i+k \cdot N \\
\quad \square R \cdot i+k \cdot N
\end{gathered}
$$

And it is known that the current $i$ is proportional to the motor torque $T_{M}$.

As can be understood from Eq. (2), the motor can be controlled based on the so-called motor current control method which is shown in Fig. 4.

In the motor current control method, the target motor current $I_{T}$, which is proportional to the motor assist torque $T_{M}$, is determined from the signal output $T$ from the torque sensor, and control is performed so that there is no difference between this target current value $I_{T}$ and the value detected through feedback from the current sensor $I_{M}$. 
In this method, the target value, which is determined by the assist characteristic based on the input of torque sensor and vehicle speed sensor, for the motor current is set so that it is equal to the vehicle speed response type derived from the signal of the vehicle speed sensor. The assist characteristic is shown in Fig. 5 (Takayuki Kifuku et al., 1997).

The typical control system of EPS device is shown in the block diagram of Fig. 6 (Masahiko Kurishige et al., 2001; Ji-Hoon Kim, 2002). The target current setting unit determines the reference current $i_{r}$ to the motor based on the driving conditions, and the controller computes the control signal which minimizes the error between $i_{r}$ and the actual current $i_{a}$.

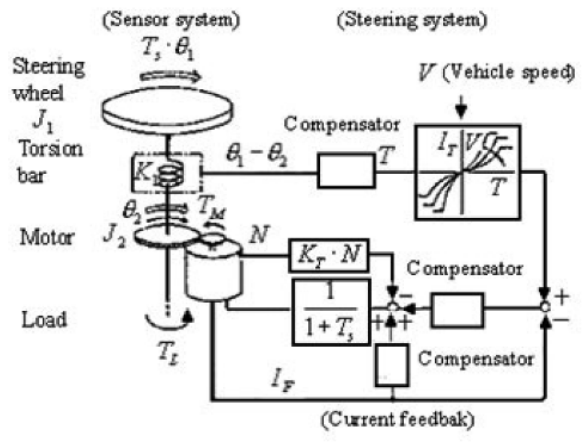

Fig. 4. Principle of motor current control

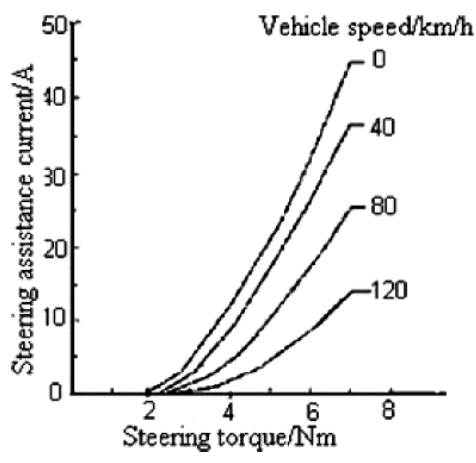

Fig. 5. Assist characteristic

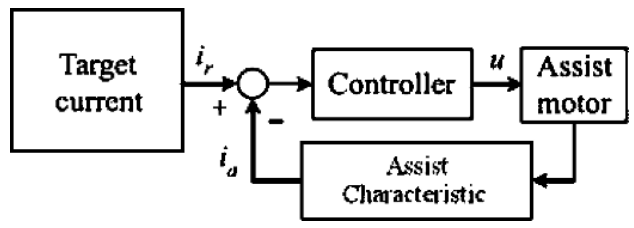

Fig. 6. Block diagram of EPS control system

\subsection{Design of EPS controller}

The EPS controller is designed based on motor current control method. The EPS controller (refer to Fig. 7) consists of an interface circuit that coordinates the signals from the various sensors, an A/D converter and a PWM unit that are all built into an one-chip microprocessor, a watchdog timer (WDT) circuit that monitors the operation of this microprocessor, the motor-drive circuit that consists of power MOSFETs in an $\mathrm{H}$ bridge circuit driven by pulse width modulation (PWM) over a $20 \mathrm{kHz}$ carrier (Ronald K. et al., 1999; Takayuki Kifuku et al., 1997; Yuji Kozaki et al., 1999; Masahiko Kurishige et al., 2001). 
The ECU conducts a search for data according to a table lookup method based on the signals input from each sensor and carries out a prescribed calculation using this data to obtain the assist force.

In addition, trouble diagnosis for the sensors and the microprocessor is also carried out. When a problem is detected, power to the motor is interrupted, an indicator lamp illuminates, and the problem condition is memorized. Then this problem mode flashes on a display as necessary (International Rectifier Application Notes; Geoffrey Walker, 1998; Jeff Burns et al., 2000; Sergio Fissore et al., 2000).

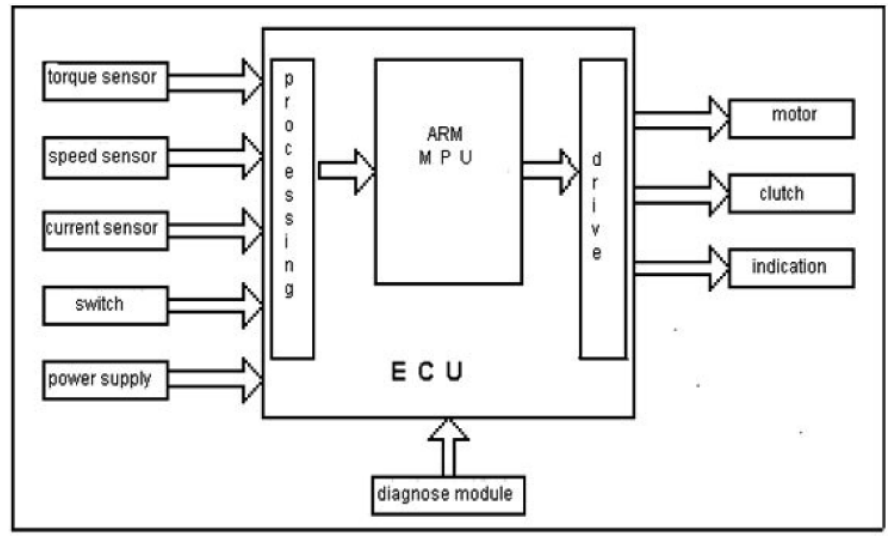

Fig. 7. Framework of EPS controller

\section{TESTS AND ANALYSIS}

On the basis of the motor control strategy and design of EPS controller, full-car tests are performed. The framework of test system is shown in Fig. 8.

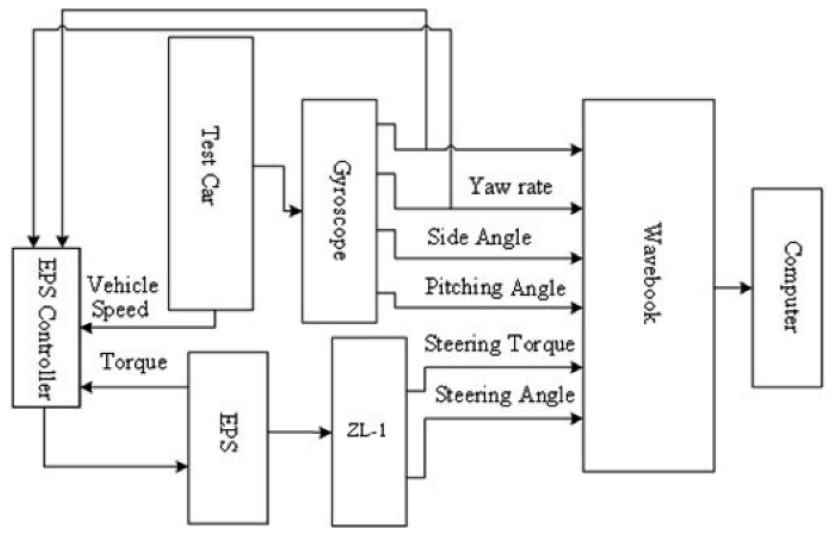

Fig. 8. Test system 
The test system is made up of the following parts. (1) sensors: ZL-1 steering parameters test system, vehicle speed sensor, VG400 gyroscope (Grossbow co.), (2) controller: Electric Control Unit with ARM S3C44B0X, the main input signals include torque signal, vehicle speed signal and motor current signal, (3) signal collection system: WaveBook $512 \mathrm{H}$, computer and DASYLab8.0.

\subsection{Steering handiness test}

When the EPS system works normally and the vehicle speed is zero, rapidly turn the steering wheel to an angle about $600^{\circ}$ and keep still, the angle-torque curve and angle-current curve which is shown in Fig. 9 can be obtained.

Also, when the EPS system works normally and the vehicle speed is $5 \mathrm{~km} / \mathrm{h}$, rapidly turn the steering wheel to a angle about $600^{\circ}$ and keep still, the angle-torque curve and angle-current curve which is shown in Fig. 10 can be obtained.

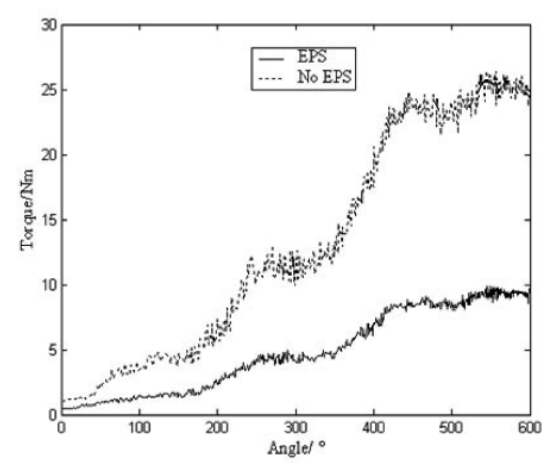

(a) Angle-torque curve

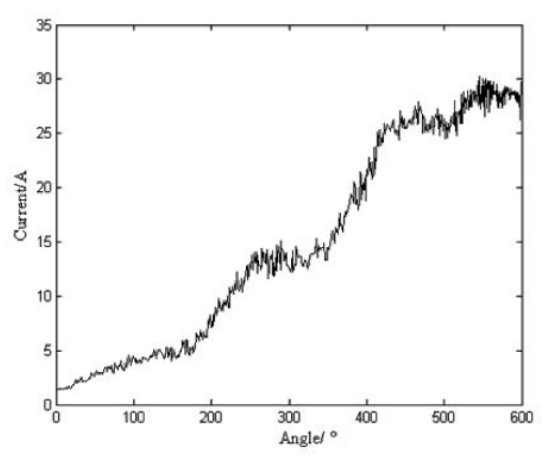

(b) Angle-current curve

Fig. 9. Steering with zero vehicle speed condition

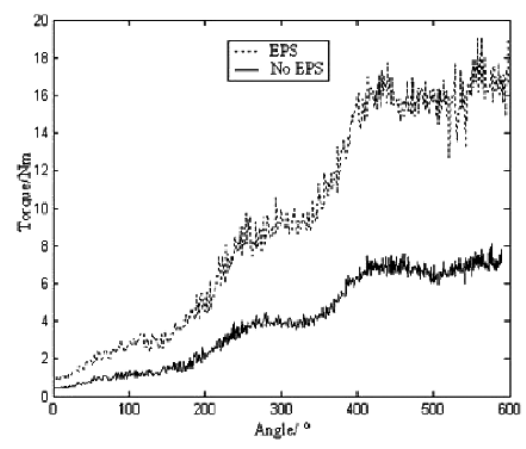

(a) Angle-torque curve

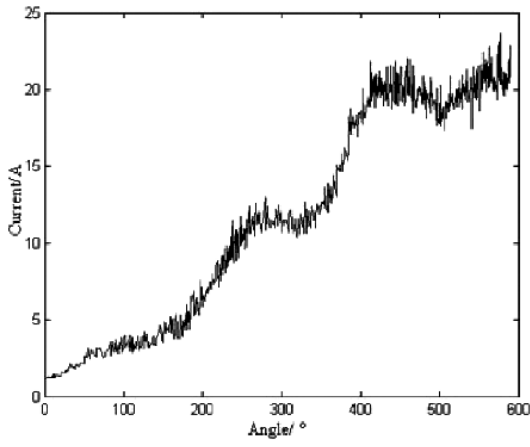

(b) Angle-current curve

Fig. 10. Steering with $5 \mathrm{~km} / \mathrm{h}$ vehicle speed condition 
And, the detailed comparation is listed as Table 1, when there is Esther handiness is obvious. When the vehicle speed is zero, the handiness is increased $69.5 \%$, and $5 \mathrm{~km} / \mathrm{h} 56.3 \%$.

Table 1. Steering handiness test efficiency

\begin{tabular}{|l|l|c|c|}
\hline Condition & Item & No EPS & EPS \\
\hline Zero speed & Peak torque/Nm & 26.42 & 9.658 \\
\hline & Average torque/Nm & 14.11 & 4.308 \\
\hline & Handiness target & \multicolumn{2}{|c|}{3.275} \\
\hline $5 \mathrm{~km} / \mathrm{h}$ speed & Peak torque/Nm & 19.11 & 7.8 \\
\hline & Average torque $/ \mathrm{Nm}$ & 8.713 & 3.806 \\
\hline & Handiness target & \multicolumn{2}{|c|}{2.289} \\
\hline
\end{tabular}

\subsection{Step input test}

When the EPS system works normally and the vehicle speed is respectively zero and $5 \mathrm{~km} / \mathrm{h}$, rapidly turn the steering wheel to a angle and keep still for several seconds, the corresponding motor current response curves are shown in Fig. 11.

It's revealed that the motor current response is rapid and can rapidly achieve stabilization, and can fully meet the demand of real time.

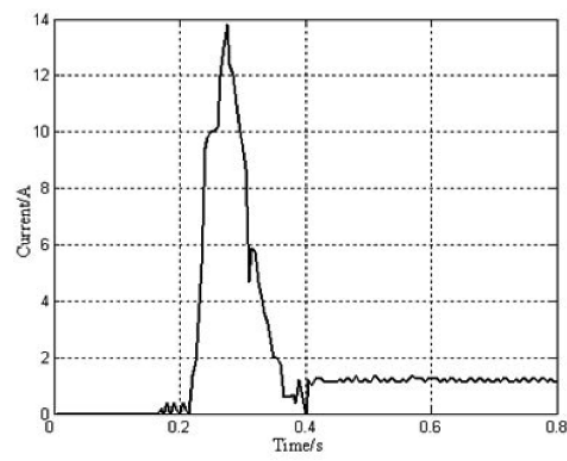

(a) Currnet response(zero)

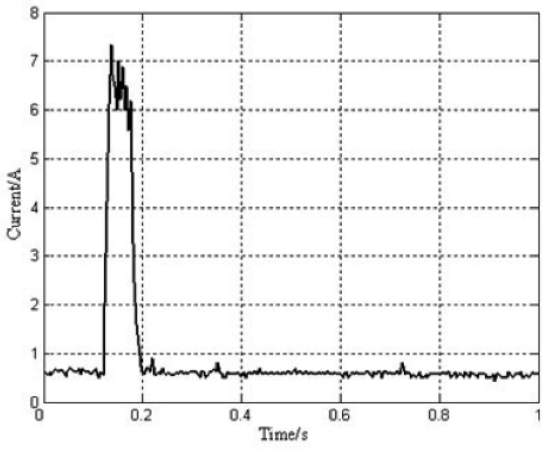

(b) Currnet response $(5 \mathrm{~km} / \mathrm{h})$

Fig. 11. Motor current response

\section{CONCLUSIONS AND DISCUSSIONS}

The results show that the EPS controller designed is stable and credible, and can meet the requirements of steering performance.

The demands for faster speed, higher quality, and reduced power requirements in vehicles are continually increasing. In order to respond to these demands, research and development is under way on the application of 
electronic control with the aim of further improving functions and performance. Features that are being proposed include the introduction of intelligent control strategy and the application of power steering, which responds to the driving environment by varying the assist amount in accordance to fit the sensitivities of human operators.

\section{ACKNOWLEDGEMENTS}

The authors acknowledge support of Zhu Yongjun in designing the steering tests and the controller. Chen Dayu provided the matlab/simulink support. This work was supported by National Natural Science Foundation of China under Grant No. 50475121 and Jiangsu Provincial High-tech Project of China under Grant No. BG2004025. The contents of this paper reflect the view of the authors who are responsible for the facts and accuracy of the data presented in herein. The contents do not necessarily reflect the official views or policies of Jiangsu University. This paper do not constitute a standard, specification or regulation.

\section{REFERENCES}

Geoffrey Walker. A motor controller for the solar car project.The university of Queensland, 1998

International Rectifier, IR2110 datasheet, Data sheet NO, PD-6.011E

International Rectifier. Bootstrap Component Selection For Control IC's, DT98-2a

International Rectifier. HV Floating MOS-Gate Driver IC's, AN978

Jeff Burns, Suresh Chengalva. Integrated motor drive unit-A mechatronics packaging concept for automotive electronics, SAE paper, 2000-01-0132

Jiang Haobin, Zhao Jingbo, Chen Long. Hardware design and experiment research of automotive electric power steering system, The 3rd China-Japan Conference on Mechatronics 2006 Fuzhou, 68-71

Ji-Hoon Kim, Jae-Bok Song. Control logic for an electric power steering system using assist motor, Mechatronics, 2002, 12:447-459

Masahiko Kurishige et al. Static Steering-Control System for Electric-Power Steering, TECHNICAL REPORTS, 2001

Massachusetts Institute of Technology, Development of machanical engineering

Ronald K. Jurgen. Automotive electronics handbook, Second edition, McGraw-Hill, Inc, 1999

Sergio Fissore, Leigh Cormie. Development of an electronic power assisted steering package, SAE paper, 2000-01-3069

Takayuki Kifuku, Shun'ichi Wada. An Electric Power-Steering System, TECHNICAL REPORTS, 1997

Toshinori Tanaka, Motors for electric power steering, TECHNICAL REPORTS, 2003

Yuji Kozaki, Shozo Sekiya et al. Electric Power Steering (EPS), Motion \& Control, No. 6, 1999 